\title{
Design of Optimal Material Properties for Structures Composed of Nonlinear Material
}

\author{
Martin P. Bendsøe \\ Mathematical Institute, \\ The Technical University of Denmark, \\ DK-2800 Lyngby, Denmark \\ José M. Guedes \\ IDMEC, \\ Instituto Superior Tecnico, \\ Av. Rovisco Pais, \\ P-1096 Lisboa Codex, Portugal. \\ Sheldon Plaxton \\ and \\ John E. Taylor \\ Department of Aerospace Engineering, \\ University of Michigan, \\ Ann Arbor, MI 48109-2140, USA
}

\begin{abstract}
.
Recent results on the design of material properties in the context of global structural optimization provide, in analytical form, a prediction of the optimal material tensor distributions for two or three dimensional continuum structures. The model developed for that purpose is extended here to cover the design of a structure and associated material properties for a system composed of a generic form of nonlinear softening material. As was established in the earlier study on design with linear materials, the formulation for combined 'material and structure' design with softening materials can be expressed as a convex problem. However, in contrast to the case with linear material, the optimal distribution of material properties predicted in the nonlinear problem depends on the magnitude of load. Computational solutions are presented for an example problem, showing how the optimal designs vary with different values assigned to data that fix the load and material parameters.
\end{abstract}

\section{Introduction.}

The purpose here is to treat, in analytical form, the design problem for simultaneous prediction of material properties and structural layout. In the present approach, this is accomplished simply by considering design to be characterized in the formulation via a free parametrization of the rigidity tensor of material. Formulations of this kind have been demonstrated recently for structures composed of linearly elastic material, in both a single purpose and multiple purpose design context 4,5 . In these studies the rigidity tensor is allowed to range over all positive, semidefinite tensors, and the design resource (or total cost) is measured through invariants of the tensor. The objective was taken to be 'design for minimum compliance'. Within this formulation a material optimization problem can be identified. and thus the optimal lncal form of the material tensor can be derived. Once the optimal local material properties are determined, the original design problem can be expressed as a simpler equivalent design problem statement involving only the global distribution of resource. In this way the problem size is reduced considerably. For a single loading condition this auxiliary problem takes on a simple form, one similar to that of a variable thickness sheet design problem.

In the developments to follow we will describe an extension of this free material design formulation to the design of a structure composed of a generic form of nonlinear softening material. The relevant mechanics is represented in the new formulation in terms of a generalized complementary energy principle developed recently for modelling the equilibrium analysis of such structures 14. For present purposes the design objective is likewise based

Copyright (c) 1994 by John E. Taylor. Published by the American Institute of Aeronautics, Inc. with permission. 
on complementary energy. Net material properties of the softening medium reflect a superposition of properties associated with each of a number of material constituents, and the collection of these properties, expressed through the rigidity tensors for each of these constituents, provides the problem with a set of design parameters. Analytical forms for the optimal material tensor and the global distribution of material can be derived, in much the same way as was indicated above for design with linear materials, and thus the design parameters can be removed from the problem. The reduced problem is then an equilibrium only problem, albeit with a nonlinear and non-smooth (optimal) complementary energy functional. Alternatively, by solving analytically only for the optimal local properties, the resulting reduced problem is a smooth and convex problem combining equilibrium analysis and the determination of the optimal distribution of bulk resource. This problem is tractable, and a computational example is presented to show the form of results predicted for optimal material distribution.

The work presented in this paper represents a natural extension of the recent developments on simultaneous design of material and structure [ see e.g. 4, 5]. It constitutes as well a natural progression of developments in modelling for optimal design with advanced materials, and from treatments of topology design using homogenization modelling (see, e.g., the collection of papers in 6,10 ). For models that employ the homogenization modelling for design parametrization, the optimal local material parameters can be related directly to a suitable microstructure, as demonstrated in 1,8 . In the context of the present free design parametrization, a different form of 'local structure' is required for the realization of material tensors. Examples of microstructures suitable for this purpose are described in 9,13 . These forms of local structure are not unique, nor are they necessarily of significance here other than to establish the quality of 'realizability'.

\section{Problem statement.}

As indicated in the introduction, availability of an extremum problem formulation for the analysis part of the problem is what makes it possible to treat the design of nonlinear materials conveniently. The type of formulation used in the following development, which amounts to a generalized form of complementary energy principle, is presented in detail in 14 . It is described briefly here to set the stage for the subsequent extension to cover design. The portrayal of a general form of nonlinear softening material relies on a feature in the model that has total stress expressed via a superposition of an arbitrary number of independent (constituent) fields. Each such constituent field is represented to be arbitrarily heterogeneous and anisotropic, and constituent stresses may be constrained to lie within a limiting surface. Overall material properties are determined through the model, once the parameters governing each of the the constituent fields are specified as data.

The formulation for equilibrium analysis is stated here in terms of stress fields. With the superposition of $\mathrm{P}$ softening components and one strictly linear basis component to make up the total stress, the problem has the form:

$$
\begin{aligned}
\min _{\sigma^{p}, \gamma, \alpha} & \left\{\frac { 1 } { 2 } \int _ { \Omega } \left(F_{i j k l}^{-1} \gamma_{i j} \gamma_{k l}+\right.\right. \\
& \left.\left.\sum_{p=1}^{p} E_{i j k l}^{p-1} \sigma_{i j}^{p} \sigma_{k l}^{p}\right) d \Omega\right\}
\end{aligned}
$$

subject to:

$$
\begin{aligned}
& \bar{\alpha}-\alpha \leq 0 \\
& \operatorname{div}\left(\gamma_{i j}+\sum_{p=1}^{P} \sigma_{i j}^{p}\right)+\alpha f=0 \\
& \left(\gamma_{i j}+\sum_{p=1}^{p} \sigma_{i j}^{p}\right) \cdot n=\alpha t \text { on } \Gamma_{\mathrm{T}}, \\
& \sigma^{p} \in \mathbf{K}_{p}, p=1, \ldots, P
\end{aligned}
$$

Here $E_{i j k l}^{p}$ are the rigidity tensors for the $\mathrm{P}$ softening components and $F_{i j k l}$ is the rigidity tensor for the strictly linear component. Factor $\alpha$ provides for the description of loads in the form of proportional loading. The stresses for the softening components are denoted $\sigma_{i j}^{p}$. The structure is subject to body force $f$ and surface traction $t$ on part $\Gamma_{\mathrm{T}}$ of boundary $\partial \Omega$. Finally, the convex sets of admissible stresses $\sigma_{i j}^{p}$ for the softening components are denoted by $\mathbf{K}_{p}$.

Problem (A) is written for a given material, and for the analysis problem which it models the 
combined rigidity tensors, and the information that serves to define sets $\mathbf{K}_{p}$ altogether comprise the data which govern overall material properties. For the 'design of material properties' problem to be considered below, one or more of these material property tensors are treated as design variables. Also, since the solution of (A) implies that $\alpha=\bar{\alpha}$, this result will be used bellow.

Following the recent studies on design of optimal material parameters cited in the introduction, it is natural to consider here the extension covering design of the nonlinear material. Using the rigidity tensors as free design variables, this design problem has the form:

$$
\begin{aligned}
& \inf _{E^{p}, F} \min _{\sigma^{p}, \gamma}\left\{\frac { 1 } { 2 } \int _ { \Omega } \left(F_{i j k l}{ }^{-1} \gamma_{i j} \gamma_{k l}+\right.\right. \\
& \left.\left.\quad \sum_{p=1}^{P} E_{i j k l}^{p}-1 \sigma_{i j}^{p} \sigma_{k l}^{p}\right) d \Omega\right\} \\
& \text { subject to: } \\
& \operatorname{div}\left(\gamma_{i j}+\sum_{p=1}^{P} \sigma_{i j}^{p}\right)+\bar{\alpha} f=0 \\
& \left(\gamma_{i j}+\sum_{p=1}^{p} \sigma_{i j}^{p}\right) \cdot n=\bar{\alpha} t \text { on } \Gamma_{\mathrm{T}}, \\
& \sigma^{p} \in \mathbf{K}_{p}, p=1, \ldots, P \\
& E^{p}>0, F>0, \\
& \int_{\Omega} \Psi(F) d \Omega \leq V_{o} \\
& \int_{\Omega} \Psi\left(E^{p}\right) d \Omega \leq V_{p}, p=1, \ldots P
\end{aligned}
$$

Here the design is to be optimal with respect to all positive definite rigidity tensors, and 'material resource' is measured in terms of invariants (symbolized by $\Psi$ in problem statement (P)) of these tensors. In the statement $(P)$ we take the supremum over the rigidity tensors, as we are using a stress based formulation; this is inherent to the analysis case under study. For pure displacement based formulations [see e.g., ${ }^{5}$ ], the design optimization can be performed over all positive semi-definite rigidity tensors. The difference in approach is not reflected in the solution, but rather relates to the matter of having the problem expressed in a well posed form.
We choose here to use either the trace or the Frobenius norm to measure resource for all tensors in the formulation, and this means that the invariants $\Psi(F) ; \Psi\left(E^{p}\right)$ in (P), hereafter represented as 'resource densities $\rho$ ', are given as:

$$
\rho_{t r}(F)=F_{i j i j} ; \rho_{t r}\left(E^{p}\right)=E_{i j i j}^{p}, p=1, \ldots, P
$$

for the trace measure and

$$
\begin{aligned}
& \rho_{F}(F)=\sqrt{F_{i j k l} F_{i j k l}} \\
& \rho_{F}\left(E^{p}\right)=\sqrt{E_{i j k l}^{p} E_{i j k l}^{p}}, p=1, \ldots, P
\end{aligned}
$$

for the Frobenius norm. Note that these measures are homogeneous of degree one. Thus comparing to the conventional 2D problem for the design of material distribution in a sheet (where total cost is proportional to the volume of material), the above 'cost measures' correspond in their role to the sheet thickness.

In the formulation above it is assumed that the sets $K_{p}$ of admissible softening components

$\sigma^{\mathrm{P}}$ of the total stress are design independent.. Thus the solution to problem (P) predicts the optimal distribution of rigidities within these specified softening limits (Optimal design with the limits themselves as design variables is treated for arbitrary trussed structures in ${ }^{15}$ ).

In the case of truss structures modelled as above, design for maximum load carrying capacity using member cross-sectional areas as design variables has been studied in ${ }^{7}$ for the case of an elasto-plastic formulation. Truss design for the general softening material is reported in 15,17 .

\section{Analytical reduction of the problem.}

For the case where no softening constituents are present, problem (P) is precisely a complementary energy based formulation of the minimum compliance design problem with free material design; this problem is described in detail in 5 . Along the lines of the modelling used in that study, parameters that describe the structure are now divided into two groups, namely those parameters that measure the amount of resource assigned to each point of the domain, and a second set that delineates how this resource is used to form the local material tensor. 
This provides for the following multi-level formulation of the problem:



$$
\begin{aligned}
& \left\{\begin{aligned}
\min _{\sigma^{p}, \gamma}\left[\frac { 1 } { 2 } \int _ { \Omega } \left(F_{i j k l}^{-1} \gamma_{i j} \gamma_{k l}+\right.\right. \\
\left.\left.\sum_{p=1}^{p} E_{i j k l}^{p-1} \sigma_{i j}^{p} \sigma_{k l}^{p}\right) d \Omega\right]
\end{aligned}\right. \\
& \text { subject to: } \\
& \operatorname{div}\left(\gamma_{i j}+\sum_{p=1}^{p} \sigma_{i j}^{p}\right)+\bar{\alpha} f=0 \\
& \left(\begin{array}{l}
\left(\gamma_{i j}+\sum_{p=1}^{p} \sigma_{i j}^{p}\right) \cdot n=\bar{\alpha} t \text { on } \Gamma_{\mathrm{T}}, \\
\sigma^{p} \in \mathbf{K}_{p}, p=1, \ldots, P
\end{array}\right.
\end{aligned}
$$

Here the statical admissibility conditions of the inner problem are independent of the design variables. Thus minimization with respect to the pointwise variation of the rigidity tensors can be represented in the form:

$$
\inf _{\substack{E^{P}>0, F>0, \Psi^{\prime}(F)=\rho_{0}, \Psi\left(E^{p}\right)=\rho_{p}}}\left\{F_{i j k l}^{-1} \gamma_{i j} \gamma_{k l}+\sum_{p=1}^{P} E_{i j k l}^{p-1} \sigma_{i j}^{p} \sigma_{k l}^{p}\right\}
$$

This characterization is consistent with the assumption of pointwise independent variation of the tensors within fixed values $\rho_{o}, \rho_{p}$ of resource. This in turn justifies minimization of the local measure in (P2) at each point of the structure. Problem (P2) has been studied in 5 , represented there in a strain formulation, and from the results of that reference or by direct inspection we can conclude that

$$
\inf _{E>0, \Psi(E)=\rho} E_{i j k l}^{-1} \sigma_{i j} \sigma_{k l}=\frac{1}{\rho} \sigma_{i j} \sigma_{i j}
$$

for any stress field and any rigidity tensor. This result applies for both the trace and Frobenius norm measures of resource. Note that the optimal energy expression in (P3) coincides with the energy of a linearly elastic, zero-Poisson-ratio material of density $\rho$. The infimum in (P3) is not achieved, as the optimal rigidity tensor is given by:

$$
E_{i j k l}=\rho \frac{1}{\sigma_{p q} \sigma_{p q}} \sigma_{i j} \sigma_{k l}
$$

This corresponds to a singular orthotropic material, with axes of orthotropy co-aligned with the direction of principal stresses for the field $\sigma_{i j}$, and with only one non-zero eigenvalue.

With the introduction of $(\mathrm{P} 3)$ into $(\mathrm{P} 2)$, the problem (P1) can now be reduced to the convex problem:

$$
\begin{array}{r}
\inf _{\rho_{p}, \rho_{0}} \min _{\sigma^{p}, \gamma}\left[\frac { 1 } { 2 } \int _ { \Omega } \left(\frac{1}{\rho_{0}} \gamma_{i j} \gamma_{i j}+\right.\right. \\
\left.\left.\sum_{p=1}^{P} \frac{1}{\rho_{p}} \sigma_{i j}^{p} \sigma_{i j}^{p}\right) d \Omega\right]
\end{array}
$$

subject to:

$$
\begin{aligned}
& \operatorname{div}\left(\gamma_{i j}+\sum_{p=1}^{p} \sigma_{i j}^{p}\right)+\bar{\alpha} f=0 \\
& \left(\gamma_{i j}+\sum_{p=1}^{p} \sigma_{i j}^{p}\right) \cdot n=\bar{\alpha} t \text { on } \Gamma_{\mathrm{T}}, \\
& \sigma^{p} \in \mathbf{K}_{p}, p=1, \ldots, P \\
& \int_{\Omega} \rho_{o} d \Omega \leq V_{o} \\
& \int_{\Omega} \rho_{p} d \Omega \leq V_{p}, p=1, \ldots P
\end{aligned}
$$

In (P4) the energy measure for each constituent corresponds to the complementary energy of a linear elastic, zero-Poisson-ratio material of density equal to the locally assigned resource value .

In problem (P4) we can solve for the resource densities, facilitated by the fact that the statical admissibility conditions of the inner equilibrium problem are independent of the design variables, and that resource constraints are active. The resulting resource densities are:

$\rho_{0}=V_{o} \sqrt{\gamma_{i j} \gamma_{i j}} / \int_{\Omega} \sqrt{\gamma_{i j} \gamma_{i j}} d \Omega$ 


$$
\rho_{p}=V_{p} \sqrt{\sigma_{i j}^{p} \sigma_{i j}^{p}} / \int_{\Omega} \sqrt{\sigma_{i j}^{p} \sigma_{i j}^{p}} d \Omega
$$

With the insertion of this result in problem statement (P4), the equivalent but now design independent problem takes the form:

$$
\begin{gathered}
\min _{\sigma^{p}, \gamma}\left\{\frac{1}{2 V_{o}}\left[\int_{\Omega} \sqrt{\gamma_{i j} \gamma_{i j}} d \Omega\right]^{2}+\right. \\
\left.\sum_{p=1}^{p} \frac{1}{2 V_{p}}\left[\int_{\Omega} \sqrt{\sigma_{i j}^{p} \sigma_{i j}^{p}} d \Omega\right]^{2}\right\}
\end{gathered}
$$

subject to:

$$
\begin{aligned}
& \operatorname{div}\left(\gamma_{i j}+\sum_{p=1}^{p} \sigma_{i j}^{\prime \prime}\right)+\bar{\alpha} f=0 \\
& \left(\gamma_{i j}+\sum_{p=1}^{p} \sigma_{i j}^{p}\right) \cdot n=\bar{\alpha} t \text { on } \Gamma_{\mathrm{T}}, \\
& \sigma^{p} \in \mathbf{K}_{p}, p=1, \ldots, P
\end{aligned}
$$

This (convex) problem is a generalized minimum complementary energy statement that is applicable for a linear-softening material with a non-smooth energy functional, one that is not simply quadratic. However, the energy functional is homogeneous of degree two, meaning that the energy functional under proportional loading resembles the energy of an elastic-softening material with linear material components.

This problem constitutes a generalization of the classical plastic design formulations for truss structures, the extension here covering linearsoftening materials in a continuum setting. Its counterpart for truss structures with linearsoftening material can be stated in the form:

$$
\begin{gathered}
\min _{\sigma^{p}, \gamma}\left\{\left(1 / 2 V_{o}\right)\left[\sum_{i=1}^{m} a_{i} l_{i}\left|\gamma_{i}\right|\right]_{i}\right]^{2}+ \\
\left.\sum_{p=1}^{P}\left(1 / 2 V_{p}\right)\left[\sum_{i=1}^{m} a_{i} l_{i}\left|\sigma_{i}^{p}\right|\right]^{2}\right\}
\end{gathered}
$$

subject to:

$$
\begin{aligned}
& \mathbf{B}_{i j}\left(a_{i} \gamma_{i}+\sum_{p=1}^{P} a_{i} \sigma_{i}^{p}\right)=\bar{\alpha} f_{j} \\
& \left|\sigma_{i}^{p}\right| \leq \bar{\sigma}^{p}, p=1, \ldots, P
\end{aligned}
$$

This corresponds to a 'fully stressed' design formulation. In (P6), $a_{i}$ and $l_{i}$ denote the bar area and bar length, respectively, for the $i$ th truss member, and the softening constraint is represented as a simple stress bound which is symmetric with respect to tension and compression. A derivation of (P6) through convex duality arguments is presented for the linearly-elastic case, expressed via a displacements based minimum compliance formulation, in 2 .

The computational results presented in this paper are obtained using a code for smooth optimization problems, to solve examples that are interpreted in the form of the (convex and smooth) problem (P4). The smoothness is obtained at the expense of an increased number of variables.

\section{Computational example of design with softening material}

The computational results presented here demonstrate the design of material properties for an example within plane stress modelling of the continuum. The optimization problem (P4) is transformed into a finite-dimensional nonlinear programming problem statement via a finite element discretization of stresses, deformations, and resource densities. The discretiziation model used is the simplest one possible, namely triangular elements with constant values for both element stresses and densities, and with equilibrium enforced in the weak sense at element corner nodes. A sequential quadratic programming subroutine [NLPQL, in 12 ] is used to solve the inner minimization in (P4). This is coupled with an optimality criterion updating procedure applied to predict the resource density variables. An alternative and equally straightforward approach is to operate on the transformed version of (P4) directly, thus minimizing simoultaneously on stresses and resource densities. Details regarding features of the solution procedures are given in 11 .

We note here that there is considerable possibility for improving the efficiency of the computational solution procedure. Given the convexity property of problem (P4), the problem could be solved usi.lg recently developed, efficient interior point methods. The FEM model is basic and unrefined, its purpose here being simply to serve for the production of example applications of our problem formulation. Results 
from these computations show the checkerboard type patterns usually associated with equal order approximations of the equilibrium and density fields, as discussed in ${ }^{3}$ and in ${ }^{8}$. In the Figures presented below, the pattern has been smeared out using a straightforward scheme for 'density averaging at nodal locations'. Finally, we note that the FEM discretized versions of problem (P4) will exhibit extensive sparsity, which could be exploited in order to improve computational efficiency.

The example presented here is the problem of a symmetrically loaded sheet with a central hole as shown in Figure 1. The discretization for the quarter section analyzed is also shown in the figure. The material in this structure is represented by the linear stress component plus one softening constituent. The softening of the latter constituent is defined by the simple convex constraint

$\sigma_{11}^{2}+\sigma_{22}^{2}+2 \sigma_{12}^{2}-1.0 \leq 0$

The resource limit on each constituent is set at a value 0.3 times the resource value corresponding to a uniform distribution with $p=1.0$. Lower and upper bounds on $\rho$, with values 0.001 and 1.0 , are enforced as well. Results are presented for two values of the load parameter alpha, $\alpha=$ 0.1 and $\alpha=10.0$. We display the total (combined $\rho_{0}, \rho_{1}$ ) density distribution for the two load levels, and a representative principal stress plot (for the total stress state at the higher load) in Figures 2 and 3.

\section{Conclusions.}

We have demonstrated that the design of material can be extended to a general class of analysis situations encompassing structures made of elastic/softening or elastic/stiffening materials. The optimal material properties can be derived analytically, and this provides for a considerable simplification in the analysis and a commensurate reduction in problem size. The analysis applies as well in two and threedimensions, with the reduction in problem size being especially important in the threedimensional setting, particularly to render the computational problem into tractable size.

\section{Acknowledgments.}

This work was supported in part by the Danish Technical Research Council, through the Programme of Research on Computer Aided Design (MPB). The support of AGARD (MPB,
JMG), JNICT, Portugal (JMG) and the Danish Natural Sciences Research Council (JET) is also gratefully acknowledged.

\section{References.}

1 - Allaire, G.; Kohn, R.V. (1993b): "Optimal Design for Minimum Weight and Compliance in Plane Stress using Extremal Microstructures." European J. Mech. A.;1993 (to appear).

2 - Bendsøe, M.P.; Ben-Tal, A.; Zowe, J. (1993): "Optimization Methods for Truss Geometry and Topology Design." Structural Optimization (to appear).

3 - Bendsøe, M.P.; Diaz, A.; Kikuchi, N. (1993): "Topology and Generalized Layout Optimization of Elastic Structures." loc. cit. Bendsøe and Mota Soares, 1993, pp. 159-206.

4 - Bendsøe, M.P.; Diaz, A.; Lipton, R.; Taylor, J.E. (1993a): "Optimal Design of Material Properties and Material Distribution for Multiple Loading Conditions." DCAMM Report no. 469, The Danish Center for Applied Mathematics and Mechanics, The Technical University of Denmark, Lyngby, Denmark, 1993.

5 - Bendsøe, M.P.; Guedes, J.M.; Haber, R.B.; Pedersen, P.; Taylor, J.E. (1993b): "An Analytical Model to Predict Optimal Material Properties in the Context of Optimal Structural Design." J. Applied Mcch., 1993 (to appear).

6 - Bendsøe, M.P.; Mota Soares, C.A. (Eds.) (1993): "Topology Optimization of Structures." Kluwer Academic Press, Dordrecht, The Netherlands, 1993.

7 - Bendsøe, M.P.; Olhoff, N.; Taylor, J.E. (1993): "A Unified Approach to the Analysis and Design of Elasto-Plastic Structures with Mechanical Contact." In Rozvany, G.I.N. (Ed.), Optimization of Large Structural Systems, Kluwer Academic Publishers, Dordrecht, The Netherlands, 1993, pp. 697-706.

8 - Jog, C.; Haber, R.B. Bendsøe, M.P. (1993): "Topology Design with Optimized, SelfAdaptive Materials." Int. J. Num. Meth. Engng. (to appear).

9 - Milton. G.W., Cherkaev, A.V., "Materials with Elastic Tensors that Range Over the Entire Set Compatible with Thermodynamics," In Proc. Joint ASCE-ASME-SES Meet'N' (Herakovich, 
C.T. \& Duva, J.M., Eds.), June 6-9, 1993 , University of Virginia, Charlottesville, Virginia, USA, p. 342.

10 - Pedersen, P. (Ed.) (1993): "Optimal Design with Advanced Materials." Elsevier, Amsterdam, The Netherlands, 1993.

11 - Plaxton, S., Taylor, J.E. (1993): "Applications of a Generalized Complementary Energy Principle for the Equilibrium Analysis of Softening Material." Comp. Meth. Appl. Mech. Engng., 1993 (in press).

12 - Schittkowski, K. (1985): "NLPQL: A FORTRAN Subroutine Solving Constrained Nonlinear Programming Problems." Annals Oper. Res., Vol. 5, 1985, pp. 485-500.

13 - Sigmund, O. (1993): "Construction of Materials with Prescribed Constitutive Parameters: An Inverse Homogenization Problem." DCAMM Report no. 470, The Danish Center for Applied Mathematics and Mechanics, The Technical University of Denmark, Lyngby, Denmark, 1993. .

14 - Taylor, J.E. (1993a): "A Global Extremum Principle for the Analysis of Solids Composed of Softening Material." Int. J. Solids Struct., Vol. 30, 1993, pp. 2057-2069.

15 - Taylor, J.E. (1993b): "Truss Topology Design for Elastic/Softening Materials." loc. cit. Bendsøe and Mota Soares, 1993, pp. 451-467.

16 - Taylor, J.E. (1993c): "A Global Extremum Principle in Mixed Form for Equilibrium Analysis with Elastic/Stiffening Materials (A Generalized Minimum Potential Energy Principle)." J. Appl. Mech. (to appear).

17 - Taylor, J.E.; Logo, J. (1993): "Analysis and Design of Elastic/Softening Truss Structures Based on a Mixed-Form Extremum Principle." In Rozvany, G.I.N. (Ed.), Optimization of Large Structural Systems, Kluwer Academic Publishers, Dordrecht, The Netherlands, 1993, pp. 683-696.

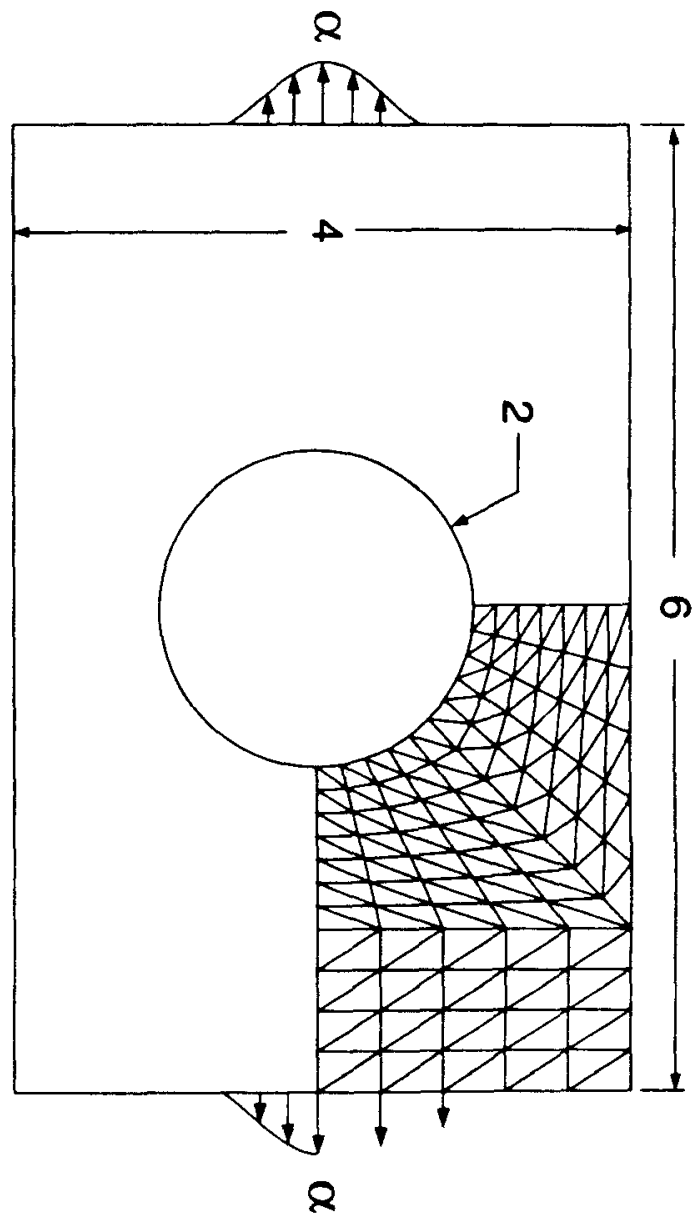

Figure 1 - Layout and discretization for design example 

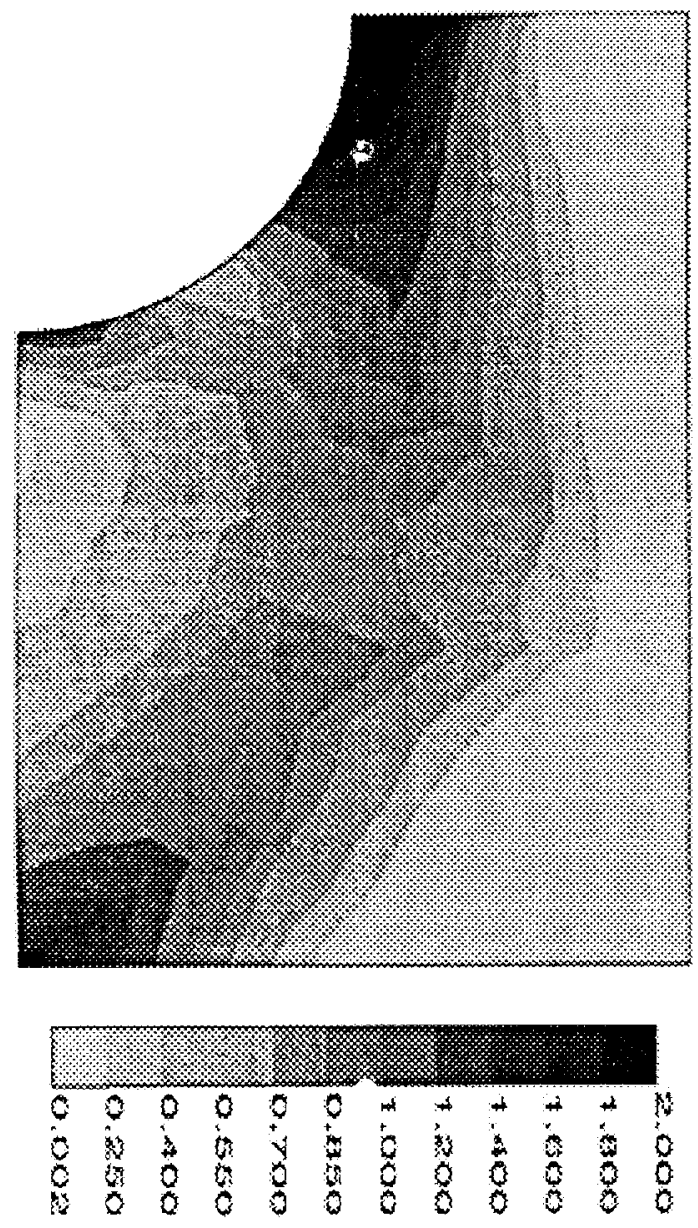

Figure 2 - Total resource distribution for load $\alpha=0.1$
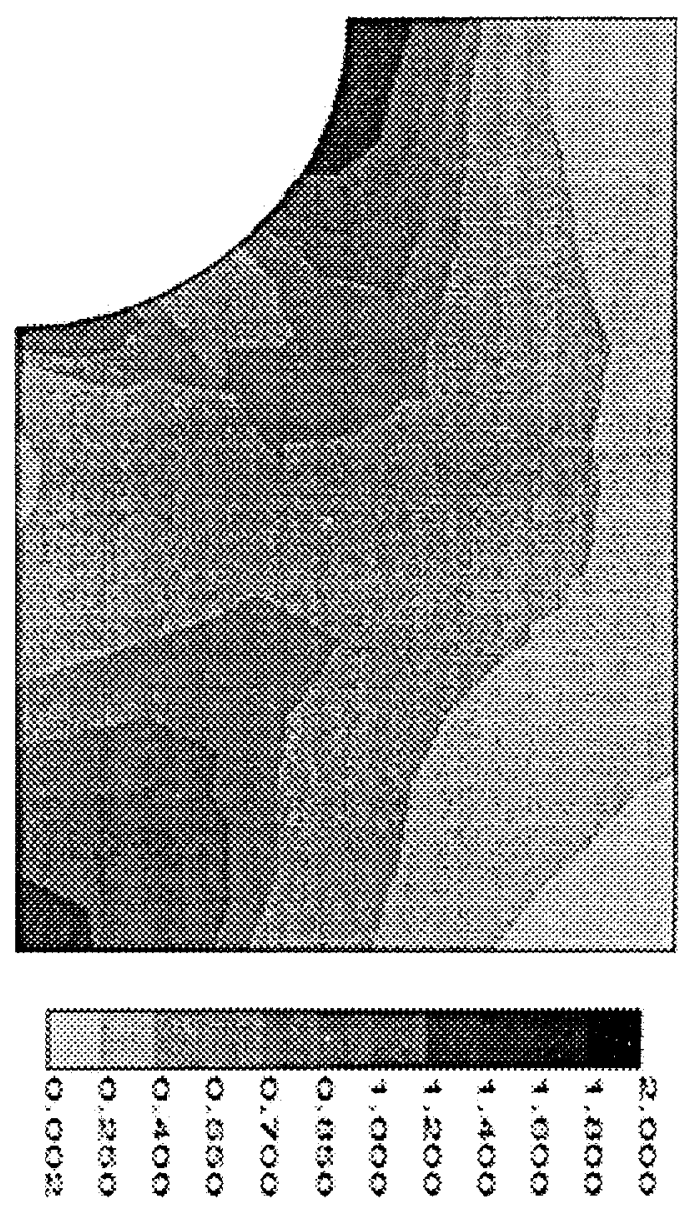

Figure 3 - Total resource distribution for load $\alpha=10.0$ 


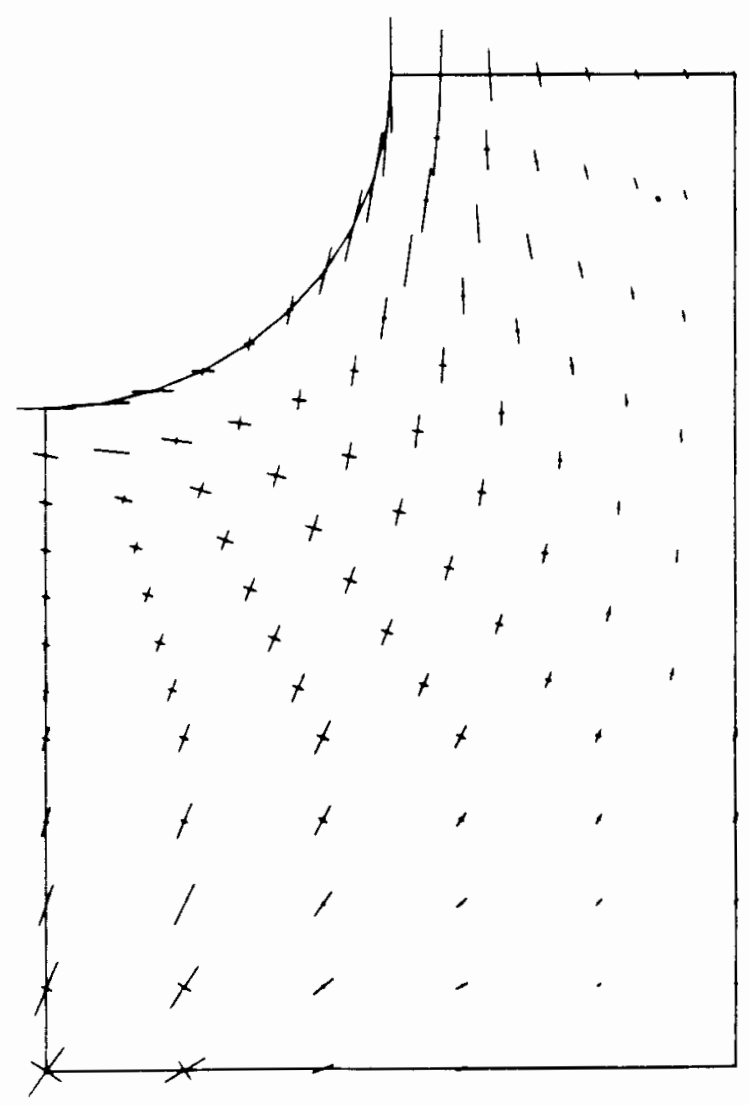

Figure 4 - Principal directions and relative magnitudes of total stress at load level $\alpha=10.0$ 